\title{
A General and Expeditious One-Pot Synthesis of Sulfoxides in High Optical Purity from Norephedrine-Derived Sulfamidites.
}

\author{
Jose L. García Ruano,* Carlos Alemparte, M. Teresa Aranda, and María M. Zarzuelo \\ Departamento de Química Orgánica, Universidad Autónoma de Madrid, Cantoblanco, 28049 - \\ Madrid, Spain
}

\section{Supporting Information}

\section{General methods.}

NMR spectra were obtained using a Bruker spectrometer (300 and $75 \mathrm{MHz}$ for ${ }^{1} \mathrm{H}$ and ${ }^{13} \mathrm{C}$, respectively) in $\mathrm{CDCl}_{3}$ solutions. Melting points were measured using a Gallenkamp apparatus in open capillary tubes. Specific rotations were measured in a Perkin-Elmer $241 \mathrm{MC}$ polarimeter. ee's of sulfoxides 6 were evaluated by HPLC using a Chiralcel OD (Chiralpak AS for cyclohexylisopropyl and cyclohexyldecylsulfoxides) column and hexane/isopropanol as an eluant.

Procedure for the preparation of sulfamidites $3 \mathbf{a}$ and $\mathbf{3 b}$.

To a stirred solution of $1.5 \mathrm{~g}(10 \mathrm{mmol})$ of $(1 R, 2 S)-(-)-$-norephedrine 1 and $4.89 \mathrm{~g}$ (40 mmol) of 4-(N,N-dimethylamino)pyridine in dry $\mathrm{CH}_{2} \mathrm{Cl}_{2}(20 \mathrm{~mL})$ at $0^{\circ} \mathrm{C}, 1.5 \mathrm{~mL}$ of $\mathrm{ClCO}_{2} \mathrm{Bn}(10.5$ mmol) were added. After $5 \mathrm{~min}$. the reaction was cooled to $-40{ }^{\circ} \mathrm{C}$ and $1.0 \mathrm{~mL}(14 \mathrm{mmol}) \mathrm{of}$ $\mathrm{SOCl}_{2}$ were added and the mixture was stirred for $15 \mathrm{~min}$. Addition of water and extraction with $\mathrm{CH}_{2} \mathrm{Cl}_{2}(3 \times 20 \mathrm{ml})$ afforded a crude mixture of 3a and 3b. 3a was isolated in $57 \%$ yield after chromatographic purification $\left(\mathrm{CH}_{2} \mathrm{Cl}_{2}\right.$ /hexane (1:1)).

For preparation of $\mathbf{3 b}$, 2,6-lutidine should be used instead of 4-( $N, N$-dimethylamino)pyridine.

Benzyl (2R, 4S, 5R)-4-methyl-5-phenyl-1,2,3-oxathiazolidine-2-oxide-3-carboxylate (3a)

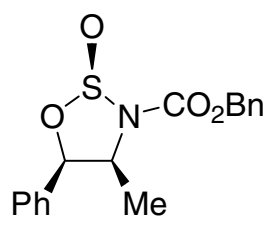

${ }^{1} \mathrm{H}$ NMR: $\delta 7.39(\mathrm{~m}, 10 \mathrm{H}), 5.77(\mathrm{~d}, J=6.4 \mathrm{~Hz}, 1 \mathrm{H}), 5.36$ and 5.27 (AB system, $J=12.1 \mathrm{~Hz}$, 2H), $4.49(\mathrm{~m}, 1 \mathrm{H}), 1.14(\mathrm{~d}, J=6.8 \mathrm{~Hz}, 3 \mathrm{H})$.

${ }^{13}$ C NMR: $\delta 151.8,134.9,133.8,128.7,128.6,128.6,128.5,128.1,126.2,90.5,68.6,54.8,16.8$. $[\alpha]_{\mathrm{D}}:-152\left(c 0.97, \mathrm{CHCl}_{3}\right)$.

Benzyl (2S, 4S, 5R)-4-methyl-5-phenyl-1,2,3-oxathiazolidine-2-oxide-3-carboxylate (3b)<smiles>[M]C1C(c2ccccc2)OB(O)N1C(=O)OCc1ccccc1</smiles>

${ }^{1} \mathrm{H}$ NMR: $\delta 7.37(\mathrm{~m}, 10 \mathrm{H}), 6.37(\mathrm{~d}, J=5.4 \mathrm{~Hz}, 1 \mathrm{H}), 5.29(\mathrm{~s}, 2 \mathrm{H}), 4.49(\mathrm{~m}, 1 \mathrm{H}), 0.87(\mathrm{~d}, J=6.5$ $\mathrm{Hz}, 3 \mathrm{H})$. 
${ }^{13}$ C NMR: $\delta 152.1,135.1,132.7,128.8,128.4,128.3,127.9,125.9,85.0,68.4,56.4,14.5$.

$[\alpha]_{\mathrm{D}}:-17\left(c 1, \mathrm{CHCl}_{3}\right)$.

m. p.: $111-112^{\circ} \mathrm{C}$.

General preparation of sulfinates 4 .

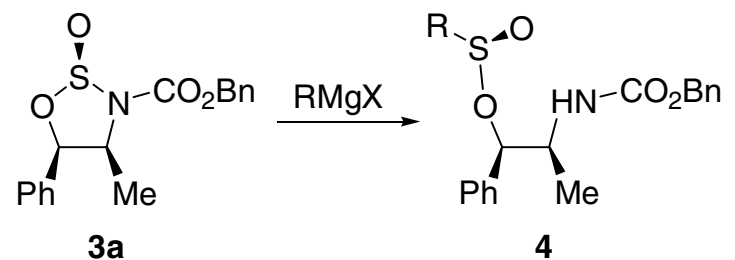

The procedure for one-pot preparation of sulfoxides (vide infra) was followed. 10-25 min after the addition of $\mathrm{RMgX}$, the reaction is quenched with $\mathrm{NH}_{4} \mathrm{Cl}$ (aq.), worked-up and purified by flash chromatography.

(1R, 2S)-2-\{[(Benzyloxy)carbonyl]amino\}-1-phenylpropyl (S)-methanesulfinate

${ }^{1} \mathrm{H}$ NMR: $\delta 7.34(\mathrm{~m}, 10 \mathrm{H}), 5.48(\mathrm{~d}, J=8.7 \mathrm{~Hz}, 1 \mathrm{H}), 5.36(\mathrm{~d}, J=2.1 \mathrm{~Hz}, 1 \mathrm{H}), 5.16$ and 5.08 (AB system, $J=12.3 \mathrm{~Hz}, 2 \mathrm{H}), 4.08(\mathrm{~m}, 1 \mathrm{H}), 2.74(\mathrm{~s}, 3 \mathrm{H}), 1.05(\mathrm{~d}, J=6.8 \mathrm{~Hz}, 3 \mathrm{H})$.

${ }^{13}$ C NMR: $\delta$ 155.9, 137.8, 136.7, 128.6 (2C), 128.5, 128.3, 128.0, 126.1, 84.5, 66.7, 51.8, 44.9, 13.7.

$[\alpha]_{\mathrm{D}}:-92\left(c 1, \mathrm{CHCl}_{3}\right)$.

m. p.: $96-97^{\circ} \mathrm{C}$.

(1R, 2S)-2-\{[(Benzyloxy)carbonyl]amino\}-1-phenylpropyl (S)-ethanesulfinate

${ }^{1} \mathrm{H}$ NMR: $\delta 7.34(\mathrm{~m}, 10 \mathrm{H}), 5.55(\mathrm{~d}, J=8.3 \mathrm{~Hz}, 1 \mathrm{H}), 5.36(\mathrm{~d}, J=2.0 \mathrm{~Hz}, 1 \mathrm{H}), 5.17$ and $5.09(\mathrm{AB}$ system, $J=12.5 \mathrm{~Hz}, 2 \mathrm{H}), 4.09(\mathrm{~m}, 1 \mathrm{H}), 2.88(\mathrm{~m}, 2 \mathrm{H}), 1.30(\mathrm{t}, J=7.5 \mathrm{~Hz}, 3 \mathrm{H}), 1.05$ (d, $J=6.9$ $\mathrm{Hz}, 3 \mathrm{H})$.

${ }^{13}$ C NMR: $\delta$ 155.9, 137.8, 136.8, 128.6 (2C), 128.5, 128.3, 128.0, 126.1, 84.7, 66.7, 51.9, 51.3, 13.8, 5.4.

$[\alpha]_{\mathrm{D}}:-96\left(c 1, \mathrm{CHCl}_{3}\right)$.

m. p.: $67-68^{\circ} \mathrm{C}$.

(1R, 2S)-2-\{[(Benzyloxy)carbonyl]amino\}-1-phenylpropyl (S)-propane-2-sulfinate

${ }^{1} \mathrm{H}$ NMR: $\delta 7.35(\mathrm{~m}, 10 \mathrm{H}), 5.64(\mathrm{~d}, J=8.7 \mathrm{~Hz}, 1 \mathrm{H}), 5.34(\mathrm{~d}, J=2.2 \mathrm{~Hz}, 1 \mathrm{H}), 5.17$ and 5.09 (AB system, $J=12.3 \mathrm{~Hz}, 2 \mathrm{H}), 4.08(\mathrm{~m}, 1 \mathrm{H}), 2.87(\mathrm{sp}, J=7.0 \mathrm{~Hz}, 1 \mathrm{H}), 1,29$ (d, $J=7.5 \mathrm{~Hz}$, $3 \mathrm{H}), 1.28(\mathrm{~d}, J=7.0 \mathrm{~Hz}, 3 \mathrm{H}), 1.06(\mathrm{~d}, J=7.0 \mathrm{~Hz}, 3 \mathrm{H})$.

${ }^{13}$ C NMR: $\delta$ 155.7, 139.2, 137.5, 128.3 (2C), 127.9, 127.7 (2C), 125.9, 84.4, 66.4, 55.5, 51.7, 13.8, 13.6.

$[\alpha]_{\mathrm{D}}:-87\left(c 0.85, \mathrm{CHCl}_{3}\right)$.

(1R, 2S)-2-\{[(Benzyloxy)carbonyl]amino\}-1-phenylpropyl (S)-2-methylpropane-2-sulfinate ${ }^{1} \mathrm{H}$ NMR: $\delta 7.31(\mathrm{~m}, 10 \mathrm{H}), 5.74(\mathrm{~d}, J=8.9 \mathrm{~Hz}, 1 \mathrm{H}), 5.32(\mathrm{~d}, 2.6 \mathrm{~Hz}, 1 \mathrm{H}), 5.15$ and $5.07(\mathrm{AB}$ system, $J=12.4 \mathrm{~Hz}, 2 \mathrm{H}), 4.06(\mathrm{~m}, 1 \mathrm{H}), 1.23(\mathrm{~s}, 9 \mathrm{H}), 1.04(\mathrm{~d}, J=6.8 \mathrm{~Hz}, 3 \mathrm{H})$. 
${ }^{13}$ C NMR: $\delta$ 155.8, 137.6, 136.7, 128.6, 128.4, 128.07, 127.9, 127.9, 126.2, 84.6, 66.5, 58.0, $51.9,21.7,13.8$.

$[\alpha]_{\mathrm{D}}:-92\left(c 1, \mathrm{CHCl}_{3}\right)$.

m. p.: $73-74^{\circ} \mathrm{C}$.

\section{$(1 R, 2 S)-2-\{[(B e n z y l o x y)$ carbonyl]amino\}-1-phenylpropyl $(S)$-benzenesulfinate}

${ }^{1} \mathrm{H}$ NMR: $\delta 7.75(\mathrm{~m}, 2 \mathrm{H}), 7.56(\mathrm{~m}, 3 \mathrm{H}), 7.37(\mathrm{~m}, 10 \mathrm{H}), 5.56(\mathrm{~d}, J=2.0 \mathrm{~Hz}, 1 \mathrm{H}), 5.50(\mathrm{~d}, J=$ $8.9 \mathrm{~Hz}, 1 \mathrm{H}), 5.19$ and 5.11 (AB system, $J=12.1 \mathrm{~Hz}, 2 \mathrm{H}), 4.1(\mathrm{~m}, 1 \mathrm{H}), 1.01(\mathrm{~d}, J=6.9 \mathrm{~Hz}, 3 \mathrm{H})$.

${ }^{13}$ C NMR: $\delta$ 155.9, 145.5, 137.9, 136.8, 132.5, 129.2, 128.6, 128.6, 128.3, 128.1, 126.3, 125.1, 84.7, 66.7, 52.0, 13.8.

$[\alpha]_{\mathrm{D}}:-116\left(c 1, \mathrm{CHCl}_{3}\right)$.

$(1 R, 2 S)$-2-\{[(Benzyloxy)carbonyl]amino\}-1-phenylpropyl $(S)$-p-methoxybenzenesulfinate

${ }^{1} \mathrm{H}$ NMR: $\delta 7.68$ and 7.03 (AA'BB' system, 4H), $7.36(\mathrm{~m}, 10 \mathrm{H}), 5.59(\mathrm{~d}, J=9.1 \mathrm{~Hz}, 1 \mathrm{H}), 5.54$ (d, $J=2.0 \mathrm{~Hz}, 1 \mathrm{H}), 5.19$ and 5.10 (AB system, $J=12.3 \mathrm{~Hz}, 2 \mathrm{H}), 4.1(\mathrm{~m}, 1 \mathrm{H}), 3.85(\mathrm{~s}, 3 \mathrm{H}), 1.01$ (d, $J=6.9 \mathrm{~Hz}, 3 \mathrm{H})$.

${ }^{13}$ C NMR: $\delta 162.9,155.9,138.2,137.1,136.8,128.6,128.2,128.0,126.9,126.3,114.6,84.1$, $66.7,55.7,52.0,13.8$.

$[\alpha]_{\mathrm{D}}:-84\left(c 1, \mathrm{CHCl}_{3}\right)$.

\section{General procedure for one-pot preparation of sulfoxides 6.}

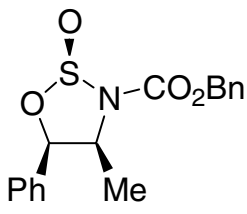

3a

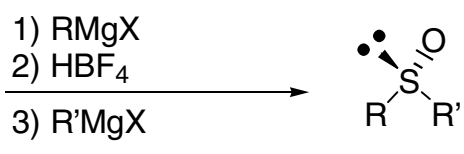

6

$\mathrm{RMgX}(0.3 \mathrm{mmol})$ is added to a solution of $\mathbf{3 a}(100 \mathrm{mg}, 0.3 \mathrm{mmol})$ in dry $\mathrm{CH}_{2} \mathrm{Cl}_{2}(3 \mathrm{~mL})$ under argon at $-78^{\circ} \mathrm{C}$. After $10-25 \min (\mathrm{TLC}), \mathrm{HBF}_{4}\left(50 \mu \mathrm{L}\right.$ of a $54 \%$ wt. solution in $\mathrm{Et}_{2} \mathrm{O}, 0.36$ mmol) is added. 5 min later, $\mathrm{R}^{\prime} \mathrm{MgX}$ ( 2.5 or 5 equiv) is added at the temperature indicated in each case (Table 1). The evolution is monitored by TLC (10-45 min). Finally, the reaction is quenched with $\mathrm{NH}_{4} \mathrm{Cl}$ (aq.) and extracted with $\mathrm{CH}_{2} \mathrm{Cl}_{2}(3 \times 5 \mathrm{ml})$. The crude mixture is purified by flash chromatography. 
Table 1. Temperature and number of equivalents for the second step.

\begin{tabular}{lllll}
\hline Entry & $\mathrm{R}$ & $\mathrm{R}$ & Temperature $\left({ }^{\circ} \mathrm{C}\right)$ & Equiv of $\mathrm{R}^{\prime} \mathrm{MgX}$ \\
\hline 1 & $\mathrm{Ph}$ & $\mathrm{Me}$ & -78 & 2.5 \\
2 & $\mathrm{Ph}$ & $i$-Pr & -78 & 2.5 \\
3 & $\mathrm{Ph}$ & Vinyl & -78 & 2.5 \\
4 & $i$-Pr & $\mathrm{Et}$ & -20 & 2.5 \\
5 & $i$-Pr & $\mathrm{Ph}$ & -20 & 2.5 \\
6 & $p$-Anisyl & $\mathrm{Et}$ & -78 & 2.5 \\
7 & $p$-Anisyl & $p$-Tol & -78 & 2.5 \\
8 & $p$-Anisyl & Vinyl & -78 & 2.5 \\
9 & $p$-Anisyl & $t$-Bu & -20 & 5.0 \\
10 & Mesityl & $p$-Tol & -20 & 5.0 \\
11 & Mesityl & Me & -20 & 5.0 \\
12 & Mesityl & Vinyl & -78 & 5.0 \\
13 & Me & Mesityl & -78 & 2.5 \\
14 & Cyclohexyl & $i$-Pr & -20 & 2.5 \\
15 & Cyclohexyl & Decyl & -20 & 2.5 \\
16 & $t$-Bu & $p$-Anisyl & r. t. & 5.0 \\
\hline
\end{tabular}

(R)-Methylphenylsulfoxide ${ }^{\mathbf{1}}$

${ }^{1} \mathrm{H}$ NMR: $\delta 7.56(\mathrm{~m}, 5 \mathrm{H}), 2.70(\mathrm{~s}, 3 \mathrm{H})$.

${ }^{13}$ C NMR: $\delta 145.3,130.7,129.0,123.1,43.6$.

$[\alpha]_{\mathrm{D}}:+137$ ( $c 1$, acetone) for a $93 \%$ ee.

Lit. ${ }^{1}[\alpha]_{\mathrm{D}}:+147.5$ (acetone).

(R)-Isopropylphenylsulfoxide ${ }^{2}$

${ }^{1} \mathrm{H}$ NMR: $\delta 7.50(\mathrm{~m}, 5 \mathrm{H}), 2.81(\mathrm{sp}, J=6.9 \mathrm{~Hz}, 1 \mathrm{H}), 1.19(\mathrm{~d}, J=6.9 \mathrm{~Hz}, 3 \mathrm{H}), 1.11(\mathrm{~d}, J=6.9$ $\mathrm{Hz}, 3 \mathrm{H})$.

${ }^{13}$ C NMR: $\delta$ 141.6, 130.8, 128.7, 124.8, 54.4, 15.7, 13.8 .

$[\alpha]_{D}:+196(c 1, \mathrm{MeOH})$ for a $93 \%$ ee.

Lit. $^{2}[\alpha]_{D}:+169.9$ (c 1.6, acetone).

(S)-Isopropylphenylsulfoxide ${ }^{2}$

$[\alpha]_{\mathrm{D}}:-198(c 1, \mathrm{MeOH})$ for a $95 \%$ ee.

(R)-Phenylvinylsulfoxide ${ }^{3}$

${ }^{1} \mathrm{H}$ NMR: $\delta 7.61(\mathrm{~m}, 2 \mathrm{H}), 7.50(\mathrm{~m}, 3 \mathrm{H}), 6.59(\mathrm{dd}, J=9.7$ and $16.6 \mathrm{~Hz}, 1 \mathrm{H}), 6.19(\mathrm{~d}, J=16.6$ $\mathrm{Hz}, 1 \mathrm{H}), 5.89(\mathrm{~d}, J=9.7 \mathrm{~Hz}, 1 \mathrm{H})$.

${ }^{13}$ C NMR: $\delta 143.3,142.9,131.1,129.3,124.6,120.5$.

\footnotetext{
${ }^{1}$ Jacobus, J.; Mislow, K. J. Am. Chem. Soc. 1967, 89, 5228.

${ }^{2}$ Sugimnoto, T.; Kokuba, T.; Miyazaki, J.; Tanimoto, S.; Okano, M. J. Chem. Soc. Chem. Commun. 1979, 402.

${ }^{3}$ Benson, S. C.; Snyder, J. K. Tetrahedron Lett. 1991, 32, 5885.
} 
$[\alpha]_{\mathrm{D}}:+333(c 0.98, \mathrm{MeOH})$ for a $95 \%$ ee.

Lit. $^{2}[\alpha]_{\mathrm{D}}(S$ config.): $-376(c$ 1, MeOH) for a $>99 \%$ ee.

(R)-Ethylisopropylsulfoxide ${ }^{4}$

${ }^{1} \mathrm{H}$ NMR: $\delta 2.76$ (sept, $\left.J=6.8 \mathrm{~Hz}, 1 \mathrm{H}\right), 2.63(\mathrm{~m}, 2 \mathrm{H}), 1.34(\mathrm{t}, J=7.5 \mathrm{~Hz}, 3 \mathrm{H}), 1.30(\mathrm{~d}, J=6.8$

$\mathrm{Hz}, 3 \mathrm{H}), 1.25$ (d, $J=6.8 \mathrm{~Hz}, 3 \mathrm{H})$.

${ }^{13}$ C NMR: $\delta 49.4,41.9,16.0,14.5,7.0$.

$[\alpha]_{\mathrm{D}}:+20\left(c 0.2, \mathrm{CHCl}_{3}\right)$

\section{(R)-Ethyl-p-methoxyphenylsulfoxide ${ }^{5}$}

${ }^{1} \mathrm{H}$ NMR: $\delta$ 7,52 and 6.99 (AA'BB' system, 4H), 3.82 (s, 3H), 2.78 (m, 2H) 1.14 (t, $J=7.3 \mathrm{~Hz}$, $3 \mathrm{H})$.

${ }^{13}$ C NMR: $\delta$ 161.9, 134.3, 126.2, 114.7, 55.6, 50.5, 6.2.

$[\alpha]_{\mathrm{D}}:+166\left(c 1.2, \mathrm{CHCl}_{3}\right)$ for a $93 \%$ ee.

\section{(R)-p-Methoxyphenyl-p-tolylsulfoxide ${ }^{6}$}

${ }^{1} \mathrm{H}$ NMR: $\delta 7.54$ and 6.93 (AA'BB' system, 4H), 7.48 and 7.23 (AA'BB' system, 4H), 3.79 (s, $3 \mathrm{H}), 2.35(\mathrm{~s}, 3 \mathrm{H})$.

${ }^{13}$ C NMR: $\delta$ 161.8, 142.6, 141.2, 129.8, 127.0, 124.7, 114.7, 55.4, 21.3.

$[\alpha]_{\mathrm{D}}:+23\left(c 1, \mathrm{CHCl}_{3}\right)$ for a $95 \%$ ee.

Lit. $^{6}[\alpha]_{\mathrm{D}}:+24$ (c 2.005, acetone).

$(\boldsymbol{R})$-p-Methoxyphenylvinylsulfoxide $\mathrm{e}^{7,8}$

${ }^{1}$ H NMR: $\delta 7.57$ and 7.01 (AA'BB' system, 4H), 6.57 (dd, $J=9.7$ and $16.6 \mathrm{~Hz}, 1 \mathrm{H}$ ), 6.19 (d, $J$ $=16.6 \mathrm{~Hz}, 1 \mathrm{H}), 5.89(\mathrm{~d}, J=9.7 \mathrm{~Hz}, 1 \mathrm{H}), 3.85(\mathrm{~s}, 1 \mathrm{H})$.

${ }^{13}$ C NMR: $\delta$ 162.2, 143.0, 140.8, 127.2, 120.2, 114.9, 55.5.

ee: $93 \%$

(R)-tert-Butyl-p-methoxyphenylsulfoxide ${ }^{5}$

${ }^{1}$ H NMR: $\delta 7.51$ and 6.99 (AA'BB' system, 4H), 3.85 (s, 3H), 1.15 (s, 9H).

${ }^{13}$ C NMR: $\delta$ 162.1, 131.0, 127.9, 114.0, 55.6, 55.5, 22.8.

$[\alpha]_{\mathrm{D}}:+116\left(c 0.9, \mathrm{CHCl}_{3}\right)$ for a $76 \%$ ee.

\footnotetext{
${ }^{4}$ Mohammed, M. S. Iraqi J. Sci. 1990, 30, 455.

${ }^{5}$ Maccagnani, G.; Taddei, F. Boll. Sci. Fac. Chim. Ind. Bologna 1965, 23, 381.

${ }^{6}$ Andersen, K. K.; Gaffiel, W.; Papanikolaou, N. E.; Foley, J. W.; Perkins, R. I. J. Am. Chem. Soc. 1964, $86,5637$.

${ }^{7}$ Bader, A.; Wuensch, J. R. Macromolecules 1995, 28, 3794.

${ }^{8}$ It was isolated partially impurified with ca. $10 \%$ of both di-p-methoxyphenylsulfoxide and divinylsulfoxide.
} 


\section{(R)-Mesityl-p-tolylsulfoxide ${ }^{9}$}

${ }^{1} \mathrm{H}$ NMR: $\delta 7.29$ and 7.20 (AA'BB' system, 4H), 6.84 (s, 2H), 2.40 (s, 6H) 2.35 (s, 3H), 2.26 (s, $3 \mathrm{H})$.

${ }^{13}$ C NMR: $\delta$ 142.0, 141.2, 139.9, 139.7, 136.7, 130.7, 129.5, 124.5, 21.2, 21.1, 19.3.

$[\alpha]_{D}:+273\left(c 1, \mathrm{CHCl}_{3}\right)$ for a $97 \%$ ee.

Lit. $^{6}[\alpha]_{\mathrm{D}}(S$ config. $):-279\left(c 0.953, \mathrm{CHCl}_{3}\right)$.

(R)-Mesitylmethylsulfoxide ${ }^{10}$

${ }^{1} \mathrm{H}$ NMR: $\delta 6.84$ (s, 2H), 2.83 (s, 6H) 2.53 (s, 3H), 2.26 (s, 3H).

${ }^{13}$ C NMR: $\delta 141.1,137.9,136.1,131.0,38.5,21.0,18.9$.

$[\alpha]_{\mathrm{D}}:+243\left(c 1, \mathrm{CHCl}_{3}\right)$ for a $97 \%$ ee.

(S)-Mesitylmethylsulfoxide ${ }^{\mathbf{1 0}}$

$[\alpha]_{\mathrm{D}}:-241\left(c 1, \mathrm{CHCl}_{3}\right)$ for a $98 \%$ ee.

Lit. ${ }^{10}[\alpha]_{\mathrm{D}}:-390\left(c 1, \mathrm{CHCl}_{3}\right)$.

(R)-Mesitylvinylsulfoxide ${ }^{11}$

${ }^{1} \mathrm{H}$ NMR: $\delta 6.84(\mathrm{~s}, 2 \mathrm{H}), 6.62(\mathrm{dd}, J=9.9$ and $16.5 \mathrm{~Hz}, 1 \mathrm{H}), 6.11(\mathrm{~d}, J=16.5 \mathrm{~Hz}, 1 \mathrm{H}), 5.93(\mathrm{~d}$, $J=9.9 \mathrm{~Hz}, 1 \mathrm{H}), 2.48(\mathrm{~s}, 6 \mathrm{H}), 2.26(\mathrm{~s}, 3 \mathrm{H})$.

${ }^{13}$ C NMR: $\delta 141.7,139.3,139.2,133.6,130.9,120.1,21.1,19.0$.

$[\alpha]_{\mathrm{D}}:+589\left(c 1.1, \mathrm{CHCl}_{3}\right)$ for a $95 \%$ ee.

\section{(R)-Cyclohexylisopropylsulfoxide ${ }^{12}$}

${ }^{1} \mathrm{H}$ NMR: $\delta 2.80(\mathrm{sp}, J=7.0 \mathrm{~Hz}, 1 \mathrm{H}), 2.53(\mathrm{~m}, 1 \mathrm{H}), 2.3-1.2(\mathrm{~m}, 10 \mathrm{H}), 1.27(\mathrm{~d}, J=7.0 \mathrm{~Hz}, 3 \mathrm{H})$, $1.26(\mathrm{~d}, J=7.0 \mathrm{~Hz}, 3 \mathrm{H})$.

${ }^{13}$ C NMR: $\delta$ 55.4, 45.9, 27.2, 25.7, 25.6, 25.3, 24.5, 17.4, 13.9.

$[\alpha]_{\mathrm{D}}:-18\left(c 1.2, \mathrm{CHCl}_{3}\right)$ for a $90 \%$ ee.

Lit. ${ }^{12}[\alpha]_{\mathrm{D}}(S$ config. $):+20.5\left(c 1, \mathrm{CHCl}_{3}\right)$ for a $>98 \%$ ee.

$(R)$-Cyclohexyldecylsulfoxide ${ }^{12}$

${ }^{1} \mathrm{H}$ NMR: $\delta 2.58(\mathrm{~m}, 2 \mathrm{H}), 2.49(\mathrm{~m}, 1 \mathrm{H}), 2.10(\mathrm{~m}, 1 \mathrm{H}), 2.0-1.6(\mathrm{~m}, 6 \mathrm{H}), 1.5-1.1(\mathrm{~m}, 19 \mathrm{H}), 0.84$ $(\mathrm{m}, 3 \mathrm{H})$.

${ }^{13}$ C NMR: $\delta$ 58.9, 48.9, 31.9, 29.5, 29.4, 29.3, 29.2, 29.0, 26.5, 25.6, 25.5, 25.3, 24.9, 22.8, 22.7 , 14.2.

$[\alpha]_{\mathrm{D}}:+22\left(c 1, \mathrm{CHCl}_{3}\right)$ for a $93 \%$ ee

Lit. ${ }^{12}[\alpha]_{\mathrm{D}}(S$ config. $):-22.1\left(c 1, \mathrm{CHCl}_{3}\right)$ for a $>98 \%$ ee.

${ }^{9}$ Shimizu, T.; Kobayashi, M. J. Org. Chem. 1987, 52, 3399.

${ }^{10}$ Khiar, N.; Araújo, C. S.; Alcudia, F.; Fernández, I. J. Org. Chem. 2002, 67, 345.

${ }^{11}$ Shibata, N.; Fujimori, C.; Fujita, S.; Kita, Y. Chem. Pharm. Bull. 1996, 44, 892.

12 Annunziata, M.; Capozzi, M.; Cardellicchio, C.; Naso, F.; Spina, G.; Tortorella, P. J. Org. Chem. 2001, 66, 5933. 